\title{
On the Fixed Point Algebra of a UHF Algebra under a Periodic Automorphism of Product Type
}

\author{
By \\ Akitaka KISHIMOTO*
}

\begin{abstract}
We study the fixed point algebra $\mathfrak{a}^{\alpha}$ of a UHF algebra $\mathfrak{A}$ under a periodic automorphisin $\alpha$ of product type. We show an example of $\mathfrak{A}^{\alpha}$ which is simple and has more than two tracial states and we characterize the case where $\mathfrak{H}^{\alpha}$ has only one tracial state. Next we show that $\mathfrak{A}^{\prime \prime}$ is a UHF algebra if and only if $\mathfrak{A}$ is generated by an infinite family of mutually commuting $\alpha$-invariant type $I_{p}$ subfactors whose fixed point algebras are abelian and by a UHF subalgebra of $\mathfrak{R}^{\alpha}$ which commutes with the former (where $p$ denotes the period of $\alpha$ ).
\end{abstract}

\section{Introduction}

E. Størmer [7] showed that the even CAR algebra is isomorphic to the CAR algebra itself. The CAR algebra is the UHF algebra of type $\left(2^{n}\right)$ and the even CAR algebra is the fixed point algebra of the CAR algebra under a specific periodic automorphism with period 2 .

In this note we study the fixed point algebra $\mathfrak{A}^{\alpha}$ of a UHF algebra $\mathfrak{i}$ under a periodic automorphism $\alpha$ of product type with period $p$, where $\alpha$ is of product type if $\mathfrak{U}$ is the $C^{*}$-tensor product of finite type $I$ factors $\mathfrak{P}_{n}$ and $\alpha$ is the product of $\alpha_{n} \in$ Aut $\mathfrak{U}_{n}$. The case studied by Størmer corresponds to $p=2$ and $\mathfrak{I}_{n}$ of type $I_{2}$. In general $\mathfrak{H}^{\alpha}$ is not necessarily a UHF algebra. In Theorem 4. 4, we give several equivalent conditions that $\mathfrak{P}^{\alpha}$ is a UHF algebra. In particular, this is the case if and only if $(\mathfrak{A}, \alpha)$ is isomorphic to $\left(\mathfrak{H}_{0} \otimes \mathfrak{U}_{p}, \iota \otimes \alpha_{p}\right)$ where $\mathfrak{U}_{0}$ is a UHF algebra, $\ell$ is the identity map and $\left(\mathfrak{U}_{p}, \alpha_{p}\right)$ is the following specific example:

Let $M$ be the full $p \times p$ matrix algebra and $e_{i j}(\mathrm{i}, \mathrm{j}=1, \cdots, p)$ its matrix units. Let $\alpha$ be the periodic automorphism of $M$ with period $p$ implemented by the unitary $\exp \left(2 \pi i p^{-1} \Sigma k e_{k k}\right)$. We let $\mathfrak{A}_{p}$ be the $C^{*}$ -

Communicated by H. Araki, January 10, 1977.

* Physics Department, Kyoto University, Kyoto 606, Japan. 
tensor product of countably infinite copies of $M$ and $\alpha_{p}$ the corresponding product automorphism of $\alpha$.

The other main result in this note is the characterization of the case where $\mathfrak{A}^{\alpha}$ has a unique tracial state, given in Theorem 3.10. One of the characterizations is that $\mathfrak{P I}^{\alpha}$ contains sufficiently large UHF subalgebras in the following sense: For any $\varepsilon>0$ there exist a projection $e$ of $\mathfrak{H}^{\alpha}$ with $\tau(e)>1-\varepsilon$, a UHF subalgebra $\mathfrak{B}$ with $e$ as identity and a sequence $\left\{e_{n}\right\}$ of projections of $\mathfrak{B}$ with $\tau\left(e_{n}\right) \rightarrow \tau(e)$ as $n \rightarrow \infty$ such that any $x$ of $\mathfrak{U}^{\alpha}$ has a sequence $\left\{x_{n}\right\} \subset \mathfrak{B}$ satisfying $\left\|e_{n} x e_{n}-x_{n}\right\| \rightarrow 0$ as $n \rightarrow \infty$, where $\tau$ is the unique tracial state of $\mathfrak{A}$.

It has been shown in [6] that $\mathfrak{A}^{\alpha}$ is simple if and only if the invariant $\Gamma(\alpha)$ is equal to $Z_{p} \equiv Z / p Z$.

The three situations for $\mathfrak{Y}^{\alpha}$ mentioned above have the following mutual relations: If $\mathfrak{U}^{\alpha}$ has a unique trace, then $\mathfrak{U}^{\alpha}$ is simple (c.f. [6, Th. 2]) but the converse does not hold as is shown in Remark 3. 12. If $\mathfrak{U}^{\alpha}$ is a UHF algebra, $\mathfrak{U}^{\alpha}$ has the unique trace, as is well known, but the converse does not hold (see Remark 4.5).

\section{§ 2. Invariant $\Gamma(\alpha)$}

Let $G$ be a compact abelian group and let $\left(\mathfrak{A}_{n}, G, \alpha^{(n)}\right)$ be a sequence of $C^{*}$-dynamical systems, i.e. $\mathfrak{N}_{n}$ is a $C^{*}$-algebra with 1 and $\alpha^{(n)}$ is a continuous homomorphism of $G$ into Aut $\mathfrak{A}_{n}$. Let $\mathfrak{U}$ be the infinite $C^{*}$-tensor product of $\mathfrak{U}_{n}, n=1,2, \cdots$, and let $\alpha_{g}$ be the automorphism $\otimes \alpha_{g}{ }^{(n)}$ of $\mathfrak{i}$ for each $g \in G$. Then $(\mathfrak{A}, G, \alpha)$ is a $C^{*}$-dynamical system. The $\Gamma(\alpha)$ is defined to be the intersection of $\operatorname{Sp}(\alpha \mid \mathfrak{B})$ where $\mathfrak{B}$ runs over all non-zero $\alpha$-invariant hereditary $C^{*}$-subalgebras of $\mathfrak{H}[5]$.

For each $t \in \widehat{G}$ let $N_{t}$ be the set consisting of $n$ such that $\operatorname{Sp} \alpha^{(n)} \ni t$. Let $H$ be the set of $t$ such that the cardinality of $N_{t}$ is infinite.

Lemma 2. 1. $\Gamma(\alpha)$ contains the subgroup generated by $H$.

Proof. Let $x$ be a positive element of $\mathfrak{A}^{\alpha}$ with $\|x\|=1$. Then there are a positive integer $n$ and a positive element $x_{0}$ of $\left(\otimes_{1}{ }^{n} \mathfrak{U}_{m}\right)^{\alpha}$ with $\left\|x_{0}\right\|=1$ and $\left\|x-x_{0}\right\|<2^{-1}$. For any non-zero $y \in \bigotimes_{n+1}^{\infty} \mathfrak{A}_{m}, x y x$ does not 
vanish since $\|x y x\| \geqq\left\|x_{0} y x_{0}\right\|-\left\|x_{0} y x_{0}-x y x\right\|=\|y\|-\left\|x_{0} y x_{0}-x y x\right\| \geqq\|y\|$ $-2\left\|x-x_{0}\right\|\|y\|>0$. Thus $\operatorname{Sp}(\alpha \overline{\mid x \mathfrak{H} x})$ contains $\operatorname{Sp}\left(\bigotimes_{n+1}^{\infty} \alpha^{(m)}\right)$, in particular the subgroup generated by $H$. Now it is easy to complete the proof (c.f. Lemma 4.1 in [4]).

In the following sections we take as $\mathfrak{U}_{n}$ a finite type I factor. The existence of minimal projections of $\otimes_{1}{ }^{n} \mathfrak{U}_{m}$ in $\left(\otimes_{1}{ }^{n} \mathfrak{U}_{m}\right)^{\alpha}$ for any $n<\infty$ obviously inplies

Proposition 2. 2. $\Gamma(\alpha)$ is the subgroup generated by $H$ when $i_{n}$ are finite type I factors.

In addition we remark that $\Gamma(\alpha)$ is a closed subgroup of $\widehat{G}$ in general [5].

\section{§ 3. Fixed Point Algebra}

Let $\mathfrak{I}_{n}$ be a finite type $I_{d_{n}}$ factor $\left(d_{n} \geqq 2\right)$ and let $\alpha_{n}$ be a periodic automorphism of $\mathfrak{I}_{n}$ satisfying $\alpha_{n}{ }^{p}=\iota$ where $\iota$ is the trivial automorphism and $p$ is a fixed positive integer. Then there exist matrix units $e_{i j}$ of $\mathfrak{U}_{n}$ and a function $\varphi_{n}$ on $\mathscr{X}_{n}=\left\{1,2, \cdots, d_{n}\right\}$ into $Z_{p}=Z / p Z$ such that $\alpha_{n}$ is implemented by the unitary $\exp \left[\Sigma_{j} i 2 \pi p^{-1} \varphi_{n}(j) e_{j j}^{(n)}\right]$ where $\varphi_{n}(j)$ is any representative in $Z$ of class $\varphi_{n}(j) \in Z_{p}$.

Let $\mathfrak{N}$ be the infinite $C^{*}$-tensor product of $\mathfrak{U}_{n}, n=1,2, \cdots$, and $\alpha$ be the corresponding product automorphism of $\alpha_{n}$. Then $\alpha$ is, of course, a periodic automorphism of the UHF algebra $\mathfrak{A}$ such that $\alpha^{p}=\iota$. Now we assume that $\alpha$ has period $p$ and we want to describe the fixed point algebra which is an $A F$ algebra [1, Lemma 5.3].

Let $\mathfrak{U}(n)=\bigotimes_{1}^{n} \mathfrak{U}_{m}$ and let $\mathfrak{U}(0)=\mathbb{C} \cdot 1$. Then $\mathfrak{U}(n)^{\alpha}$ is the direct sum of at most $p$ finite type $I$ factors. We construct each factor by the following procedure [1, Lemma 5.2]: Fix $t \in Z_{p}$ and set

$$
S_{t}(n)=\left\{(i, j) \in \Pi_{1}^{n} \mathscr{X}_{m} \times \Pi_{1}^{n} \mathscr{X}_{m} ; \Sigma_{1}^{n} \varphi_{m}\left(i_{m}\right)=\Sigma_{1}{ }^{n} \varphi_{m}\left(j_{m}\right)=t\right\} .
$$

For each $(i, j) \in S_{t}(n)$ let $e(i, j)=e_{i_{1} j_{1}}^{(1)} e_{i_{2} j_{2}}^{(2)} \cdots e_{i_{n} j_{n}}^{(n)}$. Then $\{e(i, j)\}$ forms matrix units of a finite type $I$ subfactor of $\mathfrak{A}(n)^{\alpha}$ which we denote by $M_{n, t}$. Then 


$$
\mathfrak{U}(n)^{\alpha}=\bigoplus_{t \in \mathbb{Z}_{p}} M_{n, t}
$$

The embedding of $\mathfrak{A}(n)^{\alpha}$ into $M_{n+1, t}$ is as follows:

$$
\bigoplus_{j=1}^{d_{n+1}} M_{n, t-\varphi_{n+1}(j)}=\bigoplus_{s \in Z_{p}} n_{s} \cdot M_{n, t-s}
$$

where $n_{s}$ denotes the multiplicity of $M_{n, t-s}$ in $M_{n+1, t}$ : the number of $\left\{j: \varphi_{n+1}(j)=s\right\}$. We know that $\mathfrak{A}^{\alpha}$ is generated by the increasing sequence $\mathfrak{U}(n)^{\alpha}$.

Let $x_{n}(n=0,1,2, \cdots)$ be a random variable with values in $Z_{p}$ such that $x_{n}=t$ occurs with probability $n_{t} / d_{n+1}$, i.e. $P\left(x_{n}=t\right)=n_{t} / d_{n+1}$. Suppose that the family $\left\{x_{n}\right\}$ are mutually independent. For $m \leqq n$, let

$$
S(m, n)=\sum_{j=m}^{n} x_{j}
$$

Denoting by $\Gamma(\alpha)$ the invariant $\Gamma$ of the action of $Z_{p}$ on $\mathfrak{U}$ by $t \in Z_{p} \rightarrow \alpha^{t}$, we can easily show on the basis of Proposition 2.2, the following:

Proposition 3. 1. $\Gamma(\alpha)=Z_{p}$ holds if and only if for any positive integer $m$ and any $t \in Z_{p}$ there exists an $n \geqq m$ such that $P(S(m, n)$ $=t)>0$.

Now we consider a stronger condition on $\left\{x_{n}\right\}$ :

Condition 3. 2. For each positive integer $m, S(m, n)$ converges in distribution, as $n \rightarrow \infty$, to a random variable which takes each value with equal probability, i.e. $\lim _{n} P(S(m, n)=t)=p^{-1}$ for any $t \in Z_{p}$.

In other words the condition is satisfied if and only if for any nonzero $t \in Z_{p}$ and any positive integer $m$,

$$
\lim _{n}\left\langle\exp i 2 \pi p^{-1} t S(m, n)\right\rangle=\lim \prod_{m}^{n}\left\langle\exp i 2 \pi p^{-1} t x_{j}\right\rangle=0
$$

where $\langle$.$\rangle denotes the mean.$

Proposition 3. 3. If $\left\{t \in Z_{p} ; \Sigma_{n \in N_{t}} d_{n}^{-1}=\infty\right\}$ generates $Z_{p}$, then Con- 
dition 3.2 is satisfied (where $N_{t}$ is defined in section 2). In particular if $\Gamma(\alpha)=Z_{p}$ and $\left\{d_{n}\right\}$ is bounded, then Condition 3.2 is satisfied.

Proof. Let $t \in Z_{p}$ be non-zero. Then there is an $s \in Z_{p}$ with $\exp i 2 \pi p^{-1} t s \neq 1$ such that $\sum_{n \in N_{s}} d_{n}^{-1}=\infty$. Then

$$
\begin{aligned}
\left|\left\langle\exp i 2 \pi p^{-1} t S(m, n)\right\rangle\right| & \leqq \Pi\left\{1-2 d_{j}^{-1}\left(1-d_{j}^{-1}\right)\left(1-\cos \frac{2 \pi}{p}\right)\right\}^{1 / 2} \\
& \leqq \Pi\left\{1-\left(2 d_{j}\right)^{-1}\left(1-\cos \frac{2 \pi}{p}\right)\right\}
\end{aligned}
$$

where the products are taken over $\left\{j \in N_{s}: m \leqq j \leqq n\right\}$. The right hand side converges to zero as $n \rightarrow \infty$ if and only if $\sum_{i \in N_{s}} d_{j}^{-1}=\infty$. Q.E.D.

Before going into discussions of our main result in this section we first show that Condition 3. 2 does not depend on the choice of $\left(\mathfrak{U}_{n}, \alpha_{n}\right)$, $n=1,2, \cdots$. Let $\mathfrak{B}(n)$ be an increasing sequence of $\alpha$-invariant finite type $I$ subfactors of $\mathfrak{U}$ such that $\mathfrak{U}=\overline{\cup \mathfrak{B}(n)}$. Then we have

Proposition 3.4. Let $\mathfrak{U}=\overline{\cup \mathfrak{U}(\bar{n})}=\overline{\cup \mathfrak{B}(n)}$ be as above. Then there is an automorphism $\theta$ of $\mathfrak{U}$ with $\theta \circ \alpha=\alpha \circ \theta$ such that for every positive integer $n$ there exists a positive integer $m$ such that $\theta(\mathfrak{B}(n)) \subset \mathfrak{N}(m)$ and $\mathfrak{U}(n) \subset \theta(\mathfrak{B}(m))$.

The proposition implies that $\left\{x_{n}\right\}$ defined through $\mathfrak{V}(n)$ satisfies Condition 3.2 if and only if $\left\{x_{n}\right\}$ defined through $\mathfrak{B}(n)$ satisfies Condition 3. 2. Thus we have our assertion.

The proof of Proposition 3.4 is the same as that of Lemma 2.6 in [1] if we show that the unitaries $u_{i}$ and $v_{i}$ there can be chosen in $\mathfrak{U}^{\alpha}$. This will be easily shown if we prove the following lemma corresponding to Lemma 2.3 in [1].

Lemma 3.5. Let $\mathfrak{B}$ be an $\alpha$-invariant finite-dimensional subalgebra of $\mathfrak{U}$ such that $\alpha \mid \mathfrak{B}$ is inner. Then for all $\varepsilon>0$ there exist a unitary operator $u \in \mathfrak{U}^{\alpha}$ and a positive integer $n$ such that $\|u-1\|<\varepsilon$ and $u \mathfrak{B} u^{*} \subset \mathfrak{A}(n)$. 
Proof. We may assume $1 \in \mathfrak{B}$. By applying Lemma 2.3 of [1] to $\mathfrak{U}^{\alpha}=\overline{\cup \mathfrak{U}(n)^{\alpha}}$ and $\mathfrak{B}^{\alpha}$ we may assume that $\mathfrak{B}^{\alpha} \subset \mathfrak{A}\left(n_{0}\right)^{\alpha}$ for some $n_{0}$. Let $\left\{f_{i, j}^{(k)}\right\}_{k=1}^{m}$ be matrix units for $\mathfrak{B}$ such that $\left(f_{i j}^{(k)} f_{p r}^{(l)}=\delta_{k l} \delta_{j p} f_{i r}^{(k)}, f_{i j}^{(k)}=f_{j i}^{(k) *}\right.$ and $)$ $\alpha\left(f_{i j}^{(k)}\right)=\exp \left\{i\left(\psi_{k}(i)-\psi_{k}(j)\right)\right\} f_{i j}^{(k)}$ with suitable functions $\psi_{k}(k=1, \cdots, m)$. Then for any $\delta>0$ we can find an integer $n \geqq n_{0}$ and a family $\left\{g_{i j}^{(k)}\right\}$ of matrix units in $\mathfrak{A}(n)$ such that $\left\|f_{i j}^{(k)}-g_{i j}^{(k)}\right\|<\delta$ and $f_{i i}^{(k)}=g_{i i}^{(k)}$ (c.f. Lemma 1. 10 of [2]). Let

$$
g_{i j}^{\prime(k)}=p^{-1} \sum_{i=0}^{b-1} \exp \left\{i l\left(\psi_{k}(j)-\psi_{k}(i)\right\} \alpha^{\prime}\left(g_{i j}^{(k)}\right) .\right.
$$

Then $g_{i j}^{\prime(k)} \in \mathcal{U}(n), \alpha\left(g_{i j}^{\prime(k)}\right)=\exp \left\{i\left(\psi_{k}(i)-\psi_{k}^{\prime}(j)\right)\right\} g_{i j}^{\prime(k)}, f_{i i}^{(k)} g_{i j}^{\prime(k)}=g_{i j}^{\prime(k)} f_{j j}^{(k)}$ $=g_{i j}^{\prime(k)}$ and $\left\|f_{i j}^{(k)}-g_{i j}^{\prime(k)}\right\|<\delta$. If $\delta$ is sufficiently small, the partial isometry $e_{i j}^{(k)}$ obtained from the polar decomposition of $g_{i}^{\prime}(k)$, which is an element of $\mathfrak{U}(n)$, satisfies $\alpha\left(e_{i j}^{(k)}\right)=\exp \left\{i\left(\psi_{k}(i)-\psi_{k}(j)\right)\right\} e_{i j}^{(k)}$ and $\left\|f_{i j}^{(k)}-e_{i j}^{(k)}\right\|<\varepsilon$. Let $u=$ $\Sigma_{k} \Sigma_{j} e_{j 1}^{(k)} f_{1 j}^{(k)}$. Then $u$ satisfies the above conditions.

Q.E.D.

Let $\tau$ be the unique tracial state of $\mathfrak{U}$ and let $\left(\pi_{\tau}, \mathfrak{S}_{\tau}, \Omega_{\tau}\right)$ be the GNS representation of $\mathfrak{A}$ associated with $\tau$. Let $\bar{\alpha}$ be the automorphism of the factor $M=\pi_{\tau}(\mathfrak{U})$ " such that $\bar{\alpha} \circ \pi_{\tau}=\pi_{\tau} \circ \alpha$. Then it is shown by Connes $\left[2\right.$, Th. 2. 4. 1] that $M^{\bar{\alpha}}$ is a factor if and only if $\Gamma(\bar{\alpha})=\operatorname{Sp} \bar{\alpha}\left(=Z_{p}\right)$. Since $\Omega_{\tau}$ is separating, $M^{\bar{\alpha}}$ is isomorphic to $M^{\bar{\alpha}} \mid\left[M^{\bar{\alpha}} \Omega_{\tau}\right]$. Thus, as $M^{\bar{\alpha}}$ $=\pi_{\tau}\left(\mathfrak{U}^{\alpha}\right)^{\prime \prime}$, we have:

Lemma 3. 6. Let $\left(M=\pi_{\tau}(\mathfrak{U})^{\prime \prime}, \bar{\alpha}\right)$ be as above. Then $\tau$ is a factor state of $\mathfrak{U}^{\alpha}$ if and only if $\Gamma(\bar{\alpha})=Z_{p}$.

Since $\pi_{r}$ is faithful, we have that $\Gamma\left(\bar{\alpha} \mid \pi_{r}(\mathfrak{A})\right)=\Gamma(\alpha)$. Let $\mathfrak{B}$ be a non-zero $\bar{\alpha}$-invariant hereditary $C^{*}$-subalgebra of $\pi_{\tau}(\mathfrak{A})$. Then there is a projection $e$ of $M^{\bar{\alpha}}$ such that $e M e$ is the weak closure $\mathfrak{B}$ of $\mathfrak{B}$ (c.f. [5]). Since $\operatorname{Sp}(\bar{\alpha} \mid \overline{\mathfrak{B}})=\operatorname{Sp}(\bar{\alpha} \mid \mathfrak{B})$ the definitions of $\Gamma(\bar{\alpha})$ and $\Gamma(\alpha)$ imply:

Lemma 3. 7. $\Gamma(\bar{\alpha}) \subset \Gamma(\alpha)$.

Let $C\left(Z_{p}\right)$ be the space of real valued functions on $Z_{p}$. Let $T_{n}$ and $T_{n}{ }^{\prime}(n=1,2, \cdots)$ be the linear transformations on $C\left(Z_{p}\right)$ defined by 


$$
\begin{aligned}
& \left(T_{n} f\right)(t)=d_{n}^{-1} \sum_{j=1}^{d_{n}} f\left(t+\varphi_{n}(j)\right)=\left\langle f\left(t+x_{n-1}\right)\right\rangle ; \\
& \left(T_{n}^{\prime} g\right)(t)=d_{n}{ }^{-1} \sum_{j=1}^{d_{n}} g\left(t-\varphi_{n}(j)\right)=\left\langle g\left(t-x_{n-1}\right)\right\rangle .
\end{aligned}
$$

Then we have

$$
\Sigma_{\iota \in Z_{p}}\left(T_{r} f\right)(t) g(t)=\Sigma_{t \in Z_{p}} f(t)\left(T_{n}^{\prime} g\right)(t) .
$$

Lemma 3.8. There exists a one-to-one correspondence between the set of all tracial positive linear functionals $\tau^{\prime}$ of $\mathfrak{H}^{\alpha}$ and the set of all sequences $\left\{f_{n}\right\}_{n=0}^{\infty}$ of positive functions of $C\left(Z_{p}\right)$ satisfying $T_{n} f_{n}=f_{n-1}$ $(n=1,2, \cdots)$, where the correspondence is given by

$$
f_{n}(t)=\prod_{1}^{n} d_{m} \cdot \tau^{\prime}\left(f_{t}^{(n)}\right)
$$

for $M_{n, t} \neq(0)$ with $f_{t}^{(n)}$ being any minimal projection of $M_{n, t}$. Furthermore $\tau^{\prime}(1)=f_{0}(0)$ holds for any pair $\tau^{\prime}$ and $\left\{f_{n}\right\}$ which satisfy (3.1) and there exists a constant $M$ such that $\left\|f_{n}\right\|_{\infty} \leqq M$ for any $n$ and for any $\left\{f_{n}\right\}$ satisfying the above condition and $f_{0}(0)=1$.

Proof. Since $\tau^{\prime}\left(f_{t}^{(n)}\right)$ does not depend on the choice of $f_{t}^{(n)}$ by the property of the trace on $M_{n, t}$, the mapping $\tau^{\prime} \mapsto\left\{f_{n}(t) ; M_{n, t} \neq(0)\right\}$ defined by $(3 \cdot 1)$ is well-defined. The component of a projection $f_{t}^{(n-1)} \neq 0$ in $M_{n, t+s}$ is the sum of $n_{s}$ orthogonal minimal projections of $M_{n, t+s}$, which implies that $T_{n} f_{n}(t) \equiv d_{n}^{-1} \sum_{j} t_{n}\left(t+\varphi_{n}(\tau)\right)=f_{n-1}(t)$ for $\left\{f_{n}(t)\right\}$ defined by $\tau^{\prime}$ through (3.1). The equality (3.1) defines $f_{n}$ for sufficiently large $n$ and so the relations $T_{n} f_{n}=f_{n-1}$ consistently define a unique sequence $\left\{f_{n}\right\}$ through (3.1).

Conversely let $\left\{f_{n}\right\} \subset C\left(Z_{p}\right)_{+}$be such that $T_{n} f_{n}=f_{n-1}$. Let $\tau_{n}^{\prime}$ be the unique tracial positive linear functional on $\mathfrak{U}(n)^{\alpha}$ satisfying (3.1). Then $T_{n} f_{n}=f_{n-1}$ implies that $\tau_{n}{ }^{\prime} \mid \mathfrak{P}(n-1)^{\alpha}=\tau_{n-1}^{\prime}$. Hence $\left\{\tau_{n}{ }^{\prime}\right\}$ defines the unique tracial positive linear functional $\tau^{\prime}$ on $\mathfrak{A}^{a}$ such that $\tau^{\prime} \mid \mathfrak{A}(n)^{a}$ $=\tau_{n}{ }^{\prime}$.

$\tau^{\prime}(1)=f_{0}(0)$ follows from the definition.

To prove the last assertion, let $g_{n}$ be a function on $Z_{p}$ for each $n=0,1,2, \cdots$ such that $g_{n}(t)=\tau\left(e_{t}{ }^{(n)}\right)$ with $e_{t}{ }^{(n)}$ being the identity of $M_{n, t}$. In particular $g_{0}(0)=1$ and $g_{0}(t)=0$ for $t \neq 0$. Then $\left\{g_{n}\right\}$ satisfies that $T_{n}^{\prime} g_{n-1}=g_{n}(n=1,2, \cdots)$. 
If $n_{0}$ is a positive integer such that $\operatorname{Sp}\left(\alpha^{\prime} \mathfrak{A}\left(n_{0}\right)\right)=Z_{p}$, then there is $\delta>0$ such that $g_{n_{0}} \geqq \delta$. If $g_{n} \geqq \delta$, then $g_{n+1}(t)=\left\langle g_{n}\left(t-x_{n}\right)\right\rangle \geqq \delta$. Thus we know that $g_{n} \geqq \delta$ for all $n \geqq n_{0}$

Let $\left\{f_{n}\right\}$ be a sequence satisfying the condition and $f_{0}(0)=1$. Then

$$
\begin{aligned}
& \sum_{t} f_{n}(t) g_{n}(t)=\Sigma f_{n}(t)\left(T_{n}{ }^{\prime} g_{n-1}\right)(t) \\
& \quad=\Sigma\left(T_{n} f_{n}\right)(t) g_{n-1}(t)=\Sigma f_{n-1}(t) g_{n-1}(t) .
\end{aligned}
$$

Thus we know that $\Sigma f_{n}(t) g_{n}(t)=f_{0}(0)=1$. Hence $f_{n}(t) \leqq \delta^{-1}$ holds for all $t \in Z_{p}$ and all $n \geqq n_{0}$. This completes the proof.

The trivial solution of $T_{n} f_{n}=f_{n-1}$ with $f_{0}(0)=1$ is $\left\{f_{n} \equiv 1\right\}$, which corresponds to the restriction of $\tau$ to $\mathfrak{A}^{\alpha}$.

Lemma 3. 9. Let $K$ be a subset of $t \in Z_{p}$ such that

$$
\lim _{n}\left|\left\langle\exp 2 \pi i p^{-1} t S(m, n)\right\rangle\right|>0
$$

for sufficiently large $m$. Then $K$ forms a subgroup of $Z_{p}$ and the order of $K$ is the number of extremal tracial states of $\mathfrak{A}^{\alpha}$. Furthermore the central decomposition of the restriction of $\tau$ to $\mathfrak{A}^{\alpha}$ gives all extremal tracial states of $\mathfrak{U}^{\alpha}$.

Proof. For $t \in K$, let $\left\{n_{j}\right\}$ be a subsequence of positive integers such that

$$
\lim _{j}\left\langle\exp 2 \pi i p^{-1} t S\left(n_{1}, n_{j}\right)\right\rangle \neq 0 .
$$

Then $\left\{\exp 2 \pi i p^{-1} t S\left(n_{1}, n_{j}\right)\right\}_{j}$ forms a fundamental sequence in the mean of order 2 and hence of order 1 . This implies that $K$ is a group.

Let $t_{0}$ be a non-zero minimal element of $K \subset\{0,1, \cdots, p-1\}$ (which devides $p$ ) and let $\left\{n_{j}\right\}$ be as above for $t_{0} \in K$. Let $\lambda$ be the limit of $\exp 2 \pi i p^{-1} t_{0} S\left(n_{1}, n_{j}\right)$ in the mean of order 2 . Then $\lambda^{q} \equiv 1$ with $q=p t_{0}{ }^{-1}$, the order of $K$. Hence we have a random variable $S_{n_{1}}$ taking values in $Z_{q}$ identified with $\{0,1, \cdots, q-1\}$ such that $\exp 2 \pi i q^{-1} S_{n_{1}}=\lambda$. Let $\rho$ be the quotient map from $Z_{p}$ onto $Z_{q}$. Then $\rho\left(S\left(n_{1}, n_{j}\right)\right)$ converges to $S_{n_{1}}$. For any non-negative function $f \neq 0$ of $C\left(Z_{q}\right)$ let $f_{n} \in C\left(Z_{p}\right)$ be such that 


$$
\begin{aligned}
f_{n}(t) & =\left\langle f\left(\rho(t)+\rho\left(S\left(n, n_{1}-1\right)\right)+S_{n_{1}}\right)\right\rangle & & \text { if } n \leqq n_{1}-1, \\
& =\left\langle f\left(\rho(t)+S_{n_{1}}\right)\right\rangle & & \text { if } n=n_{1}, \\
& =\left\langle f\left(\rho(t)-\rho\left(S\left(n_{1}, n-1\right)+S_{n_{1}}\right)\right\rangle\right. & & \text { if } n \geqq n_{1}+1 .
\end{aligned}
$$

Then we can show that $T_{n} f_{n}=f_{n-1}$. For example when $n \geqq n_{1}+1,\left(T_{n} f_{n}\right)(t)$ $=\left\langle f\left(\rho(t)+\rho\left(x_{n-1}\right)-\rho\left(S\left(n_{1}, n-1\right)\right)+S_{n_{1}}\right)\right\rangle$ due to the independence of $\rho\left(x_{n-1}\right)$ with $S_{n_{1}}-\rho\left(S\left(n_{1}, n-1\right)\right)$. As $f \not \equiv 0, f_{n} \not \equiv 0$. Thus by Lemma 3.8 we obtain a tracial positive linear functional $\tau_{f}$ corresponding to $f$.

The transformation $S$ on $C\left(Z_{q}\right)$ defined by $(S f)(t)=\left\langle f\left(t+S_{n_{1}}\right)\right\rangle$ is not degenerate since $\left\langle\exp 2 \pi i q^{-1} t S n_{1}\right\rangle \neq 0$ for any $t \in Z_{q}$. Thus, since $f$ $\in C\left(Z_{q}\right)_{+} \rightarrow \tau_{f}$ is affine, we have an injective linear mapping from $C\left(Z_{q}\right)$ into the space of all continuous self-adjoint tracial functionals of $\mathfrak{H}^{\alpha}$, which is order-preserving.

Let $\tau^{\prime}$ be a tracial state of $\mathfrak{U}^{\alpha}$ and let $\left\{f_{n}\right\}$ be the corresponding sequence in $C\left(Z_{p}\right)$ as in Lemma 3.8. Since $\left\{f_{n}\right\}$ is uniformly bounded, we have a subsequence $\left\{m_{j}\right\}$ of $\left\{n_{j}\right\}$ such that $f_{m_{j}}(t)$ converges, say to $f^{\prime}(t)$, for each $t \in Z_{p}$. Let $m(k)$ be a subsequence of $\left\{m_{j}\right\}$ such that $S(m, m(k))$ converges in distribution, say to $S_{m}{ }^{\prime}$. Then $f_{m}(t)=\left\langle f^{\prime}(t\right.$ $\left.\left.+S_{m}{ }^{\prime}\right)\right\rangle$ follows from the property $T_{n} f_{n}=f_{n-1}$ and the independence of $\left\{x_{n}\right\}$. Since $S_{m}{ }^{\prime}+q$ and $S_{m}{ }^{\prime}$ have the same distribution due to the fact that $\left\langle\exp 2 \pi i p^{-1} t S_{m}{ }^{\prime}\right\rangle=0$ for $t \notin K$, we know that $f_{m}=f_{m} \circ \rho$. Then we can show that $\left\{f_{n}\right\}$ is obtained as in (3.2) with $f=f^{\prime} \mid K$. This implies that the space of all continuous self-adjoint tracial functionals of $\mathfrak{U}^{\alpha}$ is orderisomorphic to $C\left(Z_{q}\right)$.

Let $\delta_{s}$ be a function on $Z_{q}$ such that $\delta_{s}(t)=0$ for $t \neq s$ and $\delta_{s}(s)=1$ and let $f_{s}=\delta_{s} /\left(\delta_{s}\right)_{0}(0)$. Let $\tau_{s}$ be the tracial state corresponding to $f_{s}$. Then $\tau_{s}$ are extremal tracial states of $\mathfrak{I}^{a}$ and the following equality holds:

$$
\tau=\sum_{s \in Z_{q}}\left(\delta_{s}\right)_{0}(0) \tau_{s}
$$

since $\Sigma\left(\delta_{s}\right)_{0}(0)\left(f_{s}\right)_{n}(t)=1$ for any $t \in Z_{q}$ and $n$. The decomposition (3.3) of $\tau$ is the central decomposition of $\tau$.

Q.E.D.

Now we state our main result in this section: 
Theorem 3. 10. Let $\left(\mathfrak{U}=\otimes \mathfrak{U}_{n}, \alpha=\otimes \alpha_{n}\right)$ and $\left(M=\pi_{\tau}(\mathfrak{U})^{\prime \prime}, \bar{\alpha}\right)$ be as above. Then the following statements are equivalent:

(i) $\mathfrak{i}^{\alpha}$ has a unique tracial state;

(ii) $\tau$ is a factor state of $\mathfrak{U}^{\alpha}$;

(iii) $\Gamma(\bar{\alpha})=Z_{p}$

(iv) Condition 3.2 is satisfied;

(v) For any $\varepsilon>0$ there exist a projection $e$ of $\mathfrak{U}^{\alpha}$ with $\tau(e)>1-\varepsilon$, a UHF subalgebra $\mathcal{W}$ with $e$ as identity and a sequence $\left\{e_{n}\right\}$ of projections of $\mathfrak{B}$ with $\tau\left(e_{n}\right) \rightarrow \tau(e)$ such that any $x \in \mathfrak{M}^{a}$ has a sequence $x_{n} \in \mathfrak{B}$ satisfying $\left\|e_{n} x e_{n}-x_{n}\right\| \rightarrow 0$ as $n \rightarrow \infty$;

(vi) In (v) $\left\{e_{n}\right\}$ can be chosen so that $\left\|\left[e_{n}, x\right]\right\| \rightarrow 0$ as $n \rightarrow \infty$ for any $x \in \mathfrak{U}^{\alpha}$.

If $\Gamma(\alpha) \neq Z_{p}(=\operatorname{Sp} a)$, all the statements do not hold and hence are equivalent since in this case the center of $\mathfrak{U}^{\alpha}$ is not trivial [6, Th.2]. Hence in the following we assume $\Gamma(\alpha)=Z_{p}$.

The equivalence of (ii) with (iii) is proved in Lemma 3.6 and the equivalences of (i), (ii) and (iv) are proved in Lemma 3.9. The implication (vi) $\Rightarrow(\mathrm{v})$ is trivial.

Proof. (v) $\Rightarrow$ (i) Suppose that (v) holds for some $\varepsilon<1$. Let $\tau^{\prime}$ be a tracial state of $\mathfrak{H}^{\alpha}$. For $x \in e \mathfrak{U}^{\alpha} e$ we have

$$
\tau^{\prime}(x)=\tau^{\prime}\left(e_{n} x\right)+\tau^{\prime}\left(\left(e-e_{n}\right) x\right) .
$$

The first term of the right hand side tends to $\tau^{\prime}(e) \tau(e)^{-1} \tau(x)$ as $n \rightarrow \infty$ since $\tau^{\prime}(x)=\tau^{\prime}(e) \tau(e)^{-1} \tau(x)$ for $x \in \mathfrak{B}$ by the uniqueness of a tracial state of the UHF subalgebra $\mathfrak{B}$. The second term is smaller than $\|x\|$ $\times \tau^{\prime}\left(e-e_{n}\right)=\|x\| \tau^{\prime}(e) \tau(e)^{-1} \tau\left(e-e_{n}\right)$. Thus we have $\tau^{\prime}(x)=\tau^{\prime}(e) \tau(e)^{-1}$ $\therefore \tau(x)$ for $x \in e^{\mathfrak{Q}} \mathfrak{Q}^{\alpha} e$. For any $x$ and $y$ of $\mathfrak{A}^{\alpha}$ we have

$$
\begin{aligned}
\tau^{\prime}(\text { xey }) & =\tau^{\prime}(\text { eyxe })=\tau^{\prime}(e) \tau(e)^{-1} \tau(\text { eyxe }) \\
& =\tau^{\prime}(e) \tau(e)^{-1} \tau(x e y) .
\end{aligned}
$$

This implies that $\tau^{\prime}=\tau$ since $\mathfrak{U}^{\alpha} e \mathfrak{H}^{\alpha}$ is dense in $\mathfrak{U}^{\alpha}$ by simplicity of $\mathfrak{U}^{\alpha}$.

Q.E.D. 
Proof. (iv) $\Rightarrow$ (vi) If $g_{n}(t)=\tau\left(e_{t}^{(n)}\right)$ as in the proof of Lemma 3. 8, we have

$$
g_{n}(t)=\left(T_{n}{ }^{\prime} \cdot T_{n-1}^{\prime} \cdots T_{1}{ }^{\prime} g_{0}\right)(t)=P(S(0, n-1)=t) .
$$

Then Condition 3.2 implies that $g_{n}(t) \rightarrow p^{-1}$ as $n \rightarrow \infty$. Hence for any $\varepsilon>0$ there are $n$ and a projection $e$ of $\mathfrak{P}(n)$ such that $\tau(e)>1-\varepsilon$ and $\tau\left(e e_{\iota}{ }^{(n)}\right)=p^{-1} \tau(e)$ for all $t \in Z_{p}$. Set $g_{n}{ }^{\prime}(t)=\tau\left(e e_{t}{ }^{(m)}\right)$ for $m \geqq n$. Then $T_{m+1}^{\prime} g_{m}{ }^{\prime}=g_{m+1}^{\prime}$ and hence $g_{m}{ }^{\prime}(\cdot)$ is constant for all $m \geqq n$. Thus the AF algebra $e \mathfrak{P l}^{a} e$ is defined by the increasing sequence

$$
e \mathfrak{Y}(n)^{\alpha} e \subset e \mathfrak{N}(n+1)^{\alpha} e \subset \ldots
$$

of the finite dimensional algebras where the direct summands of each $e \mathfrak{P}(m)^{\alpha} e$ are of the same type with each other.

Now we complete the proof by applying the following lemma to the system $(e \mathfrak{A}(e, \alpha ! e \mathfrak{A} e)$.

Lemma 3. 11. Let (iN, $\alpha$ ) be as above and suppose that $M_{1, t}$ $\left(t \in Z_{p}\right)$ are isomorphic with each other and that Condition 3.2 is satisfied. Then the statement (vi) in Theorem 3. 10 holds with $e=1$.

Proof. As we have remarked above the lemma, the direct summands of $\mathfrak{N}(n)^{\alpha}$ are of the same type with each other, say of type $I_{q(n)}$. Now we shall construct a subsequence $n_{k}$ of positive integers with $n_{1}=1$, an increasing sequence of subfactors $\mathfrak{B}_{k}$ of type $I_{q\left(n_{k}\right)}$ of $\mathfrak{U}\left(n_{k}\right)^{\alpha}$ and a sequence of projections $e_{k}(k \geqq 2)$ of $\mathfrak{B}_{k}$ such that $\tau\left(e_{k}\right)>1-k^{-1}$ and $e_{k} x$ $=x e_{k} \in \mathfrak{B}_{k}$ for any $x \in \mathfrak{Q}\left(n_{k-1}\right)^{\alpha}$. If this is done, the UHF subalgebra $\mathfrak{B}=\bigcup \mathfrak{R}_{k}$ and the projections $\left\{e_{k}\right\}$ satisfy the condition in (vi) of the theorem.

Let $\mathfrak{B}_{1}$ be any full $q\left(n_{1}\right) \times q\left(n_{1}\right)$ matrix subalgebra in $\mathfrak{A}\left(n_{1}\right)^{a}$. Suppose that we have $\left\{n_{k}\right\},\left\{\mathfrak{B}_{k}\right\}$ and $\left\{e_{k}\right\}$ satisfying the above conditions for $k \leqq m$. Let $h_{l}{ }^{(t)}(s)=\tau\left(e_{t}{ }^{\left(n_{m}\right)} e_{s}{ }^{(l)}\right)$ for $l \geqq n_{m_{l}}$. Then $T_{l+1}^{\prime} h_{l}{ }^{(t)}=h_{l}{ }_{l}^{(t)}$ and so

$$
h_{l}{ }^{(t)}(s)=\left\langle h_{n_{m}}^{(t)}\left(s-S\left(n_{m}, l-1\right)\right\rangle .\right.
$$

Hence there is an $l \equiv n_{m+1}$ such that for all $s_{1}$ and $s_{2}$, 


$$
\left|h_{l}{ }^{(t)}\left(s_{1}\right)-h_{l}{ }^{(t)}\left(s_{2}\right)\right|<p^{-2}(m+1)^{-1} .
$$

Since $e_{t}{ }^{\left(n_{m}\right)}$ and $e_{s}{ }^{(l)}$ all belong to $\mathfrak{U}(l)^{\alpha} \cap \mathfrak{B}_{m}{ }^{\prime}$ whose direct summands are of the same type with each other, we have a projection $e_{l, t}$ in $\mathfrak{N}(l)^{\text {a }}$ $\cap \mathfrak{B}_{m}{ }^{\prime}$ such that $e_{l, t} \leqq e_{t}{ }^{\left(n_{m}\right)}, \tau\left(e_{t}{ }^{\left(n_{m}\right)}-e_{l, t}\right)<p^{-1}(m+1)^{-1}$ and $\tau\left(e_{l, t} e_{s}{ }^{(l)}\right)$ are independent of $s$. Let $e_{m+1}=\Sigma_{t} e_{l, t}$ and let $\mathfrak{B}_{n+1}$ be a type $I_{q(1)}$ subfactor (with 1) of $\mathfrak{A}(l)^{\alpha}$ containing $e_{l, t}\left(t \in Z_{p}\right)$ and $\mathfrak{B}_{m}$. Since $\mathfrak{P}\left(n_{m}\right)^{\alpha}$ is generated by $\mathfrak{B}_{m}$ and $\left\{e_{t}{ }^{\left(n_{m}\right)}\right\}$ we have $e_{m+1} \mathfrak{M}\left(n_{m}\right)^{\alpha} e_{m+1} \subset \mathfrak{B}_{m+1}$ and by definition we have $e_{m+1} \in\left(\mathfrak{l}\left(n_{m}\right)^{\alpha}\right)^{\prime}$. Thus we have constructed $n_{m+1}$, $\mathfrak{B}_{m+1}$ and $e_{m+1}$ satisfying the conditions. This completes the proof by induction.

Remark 3.12 Let $q$ be a positive integer such that $q$ devides $p$. Then there is an example ( $\mathfrak{A}, \alpha)$ where $\mathfrak{H}^{\alpha}$ is simple and has $q$ extremal tracial states. Let $\mathfrak{U}_{n}$ be a type $I_{n^{2}}$ factor and let $\alpha_{n}$ be the automorphism of $\mathfrak{U}_{n}$ implemented by $\exp \left\{2 \pi i p^{-1} e_{n}\right\}$ where $e_{n}$ is a one-dimensional projection of $\mathfrak{U}_{n}$ if $n$ is odd and $e_{n}$ is a $q$ times $n^{2} / 2$-dimensional projection of $\mathfrak{V}_{n}$ if $n$ is even. We consider the system $\left(\mathfrak{U}=\otimes \mathfrak{U}_{n}, \alpha=\otimes \alpha_{n}\right)$. Since $P(S(m, m+2 p)=t)>0$ for all $t \in Z_{p}$ and $m$, we have $\Gamma(\alpha)=Z_{p}$ and hence $\mathfrak{U}^{\alpha}$ is simple [6]. For any $t \in Z_{p}$,

$$
\begin{aligned}
\left|\left\langle\exp 2 \pi i p^{-1} t S(m, n)\right\rangle\right|= & \prod_{m \leqq 2 k+1 \leqq n}\left|1+(2 k+1)^{-2}\left(\exp 2 \pi i p^{-1} t-1\right)\right| \\
& \times \prod_{m \leqq 2 k \leqq n} 2^{-1}\left|1+\exp 2 \pi i p^{-1} q t\right| .
\end{aligned}
$$

This implies that $\lim _{n}\left|\left\langle\exp 2 \pi i p^{-1} t S(m, n)\right\rangle\right| \neq 0$ if and only if $t \in(p / q) Z_{p}$. Thus by Lemma 3.9 we have the assertion.

\section{§ 4. The Condition for $\mathfrak{A}^{\alpha}$ to $\mathrm{Be}$ UHF}

Keep the definitions and notations in section 3. For $t \in Z_{p}$ let $\mathfrak{P}^{\alpha}(\{t\})$ be the set of $x \in \mathfrak{U}$ with $\alpha(x)=\exp \left\{2 \pi i p^{-1} t\right\} x$ and let $\mathcal{U}$ be the unitary group of $\mathfrak{A}$.

Lemma 4. 1. The following statements are equivalent:

(i) $\mathfrak{U}^{\alpha}(\{1\}) \cap \mathfrak{U \neq \varnothing ,}$

(ii) $\mathfrak{U}^{\alpha}(\{1\}) \cap \mathfrak{U}(n) \cap \mathcal{U} \neq \varnothing$ for sufficiently large $n$,

(iii) 2 contains an $\alpha$-invariant type $I_{p}$ factor $M$ such that $M^{\alpha}$ is 
abelian,

(iv) For sufficiently large $n, \mathfrak{P}(n)$ contains an $\alpha$-invariant type $I_{p}$ factor $M$ such that $\mathfrak{H}(n) \cap M^{\prime} \subset \mathfrak{H}^{\alpha}$ and $M^{\alpha}$ is abelian, (v) $P(S(0, n)=t)=p^{-1}$ for any $t \in Z_{p}$ for sufficiently large $n$.

Proof. (iv) $\Leftrightarrow$ (v) and (iv) $\Rightarrow$ (iii) $\Rightarrow$ (i) are obvious. (i) $\Rightarrow$ (ii) follows from the fact that $\cup_{n}\left(\mathfrak{U}^{\alpha}(\{1\}) \cap \mathfrak{A}(n) \cap \mathcal{U}\right)$ is dense in $\mathfrak{U}^{\alpha}(\{1\}) \cap \mathcal{U}$. Suppose that (ii) holds. Let $u$ be a unitary in $\mathfrak{H}^{\alpha}(\{1\}) \cap \mathfrak{A}(n)$, $e$ a minimal projection of the center of $\mathfrak{U}(n)^{\alpha}$ and $M$ the algebra generated by $e_{k, l}=u^{k} e \iota^{* l}(k, l=1, \cdots, p)$. Since $e_{k, l}$ forms matrix units for $M$ and $M$ contains the center of $\mathfrak{P}(n)^{\alpha}$, it is easy to see that $M$ satisfies the condition in (iv).

Q.E.D.

Proposition 4.2. If one of the conditions in Lemma 4.1 is satisfied, then $e=1$ is possible in the statements (v) and (vi) in Theorem 3. 10.

Proof. This is easily seen from the proof (iv) $\Rightarrow$ (vi) of Theorem 3. 10 and from Lemma 4.1(v).

Lemma 4. 3. The following statements are equivalent:

(i) $\mathfrak{I}^{\alpha}(\{1\}) \cap \mathcal{U}$ contains a central sequence ;

(ii) There exists a subsequence $n_{k}$ of positive integers such that $\mathfrak{P}^{\alpha}(\{1\}) \cap \mathfrak{A}\left(n_{k+1}\right) \cap \mathfrak{A}\left(n_{k}\right)^{\prime} \cap \mathcal{U} \neq \varnothing ;$

(iii) $\mathfrak{P}$ contains a central sequence $M_{k}$ of $\alpha$-invariant type $I_{p}$ factors such that $M_{k}^{\alpha}$ are abelian;

(iv) There exists a subsequence $n_{k}$ of positive integers such that $\mathfrak{Q}\left(n_{k+1}\right) \cap \mathfrak{P}\left(n_{k}\right)^{\prime}$ contains an $\alpha$-invariant type $I_{p}$ factor $M$ rith abelian $M^{\alpha}$ satisfying $\mathfrak{P}\left(n_{k+1}\right) \cap \mathfrak{U}\left(n_{k}\right)^{\prime} \cap M^{\prime} \subset \mathfrak{P}{ }^{n}$.

(v) There exists a subsequence $n_{k}$ of posilive integers such that $P\left(S\left(n_{k}, n_{k, 1}-1\right)=t\right)=p^{-1}$ for $a n y t \in Z_{p}$ and $k=1,2, \cdots$.

Proof. (iv) $\Leftrightarrow$ (v) and (iv) $\Rightarrow$ (iii) $\Rightarrow$ (i) are obvious (where $\left\{M_{k}\right\}$ is called a central sequence if $\left\|\left[x_{k}, y\right]\right\|$ converges to zero as $k \rightarrow \infty$ for any bounded sequence $x_{k} \in M_{k}$ and any $y \in \mathfrak{U}$ ). Suppose (i) and let $u_{k}$ 
be a central sequence of unitaries of $\mathfrak{H}^{\alpha}(\{1\})$. Then for any $\varepsilon>0$ and $n$ there is a $k$ such that $\left\|u_{k}-x_{1}\right\|<\varepsilon / 2$ holds for some $x_{1} \in \mathfrak{H} \cap \mathfrak{H}(n)^{\prime}$. Further there is an $m>n$ such that $\left\|u_{k}-x_{2}\right\|<\varepsilon$ for some $x_{2} \in \mathfrak{U}(m)$ $\cap \mathfrak{P}(n)^{\prime}$. This implies that there is an $x_{3} \in \mathfrak{U}(m) \cap \mathfrak{U}(n)^{\prime} \cap \mathfrak{Q U}^{\alpha}(\{1\})$ with $\left\|\iota_{k}-x_{3}\right\|<\varepsilon$. If $\varepsilon$ is sufficiently small, the partial isometry obtained from the polar decomposition of $x_{3}$ is a unitary in $\mathfrak{H}^{\alpha}(\{1\})$. Thus we have (i) $\Rightarrow$ (ii). The proof (ii) $\Rightarrow$ (iv) is the same as that in Lemma 4. 1 .

Q.E.D.

Now we recall $\left(\mathfrak{A}_{p}, \alpha_{p}\right)$ defined in section 1 .

Theorem 4. 4. Let (U, $\left.Z_{p}, \alpha\right)$ be as above. Then the following statements are equivalent:

(i) $\mathfrak{H}^{\alpha}$ is isomorphic to $\mathfrak{A}$;

(ii) $\mathfrak{A}^{\alpha}$ is a UHF algebra;

(iii) $(\mathfrak{H}, \alpha)$ is isomorphic to $\left(\mathfrak{U}_{0} \otimes \mathfrak{H}_{p}, \iota \otimes \alpha_{p}\right)$ where $\iota$ is the trivial automorphism of a UHF algebra $\mathfrak{U}_{0}$;

(iv) One of the conditions in Lemma 4.3 is satisfied.

Proof. (i) $\Rightarrow$ (ii) is obvious. Suppose (ii). Then by Lemma 2.6 of [1] there are an increasing sequence $\mathfrak{B}(n)$ of type I subfactors of $\mathfrak{i l}^{\alpha}$ and a subsequence $m_{n}$ of positive integers such that $: \mathfrak{L}^{\alpha}=\bigcup \mathcal{U}(n)$ and $\mathfrak{I}(n)^{\alpha} \subset \mathfrak{B}(n) \subset \mathfrak{P}\left(m_{n}\right)^{\alpha}, n=1,2, \cdots$. Hence the proportionality $P(S(n$, $\left.\left.m_{n}-1\right)=s-t\right)$ of the multiplicity of $M_{n, t}$ embedded in $M_{m_{n}, s}$ as $s$ varies, is independent of $t \in Z_{p}$. This implies that $P\left(S\left(n, m_{n}-1\right)=t\right)=p^{-1}$ for any $t \in Z_{p}$. Thus we have (ii) $\Rightarrow$ (iv). If (iv) holds, we have (iii) by using Lemma 4.3 (iv). Thus we have only to show that $\mathfrak{P}_{p}{ }^{\alpha_{p}}$ is isomorphic to $\mathfrak{P}_{p}$.

For the system $\left(\mathfrak{H}_{p}, \alpha_{p}\right)$ we construct an increasing sequence $\mathfrak{B}(n)$ of type $I_{p^{n}}$ subfactors such that $\mathfrak{P}(n)^{a} \subset \mathfrak{B}(n) \subset \mathfrak{V}(n+1)^{\alpha}$. Let $\mathfrak{B}$ be a subfactor of type $I_{p^{n-1}}$ of $\mathfrak{A}(n)$ and let $e_{\iota}{ }^{(n)}, t \in Z_{p}$, be a set of distinct minimal projections of the center of $\mathfrak{A}\left(n^{\alpha}\right)$. Then $\mathfrak{A}(n)^{\alpha}$ is generated by $\mathfrak{B}$ and $\left\{e_{t}^{(n)}, t \in Z_{p}\right\}$ and $e_{t}^{(n)} e_{s}^{(n+1)}$ is a minimal projection of $\mathfrak{U}(n+1)^{\alpha}$ $\cap \mathfrak{B}^{\prime}$ for any $t$ and $s$ in $Z_{p}$. Hence there exists a subfactor $\mathfrak{B}_{1}$ (of type $\left.I_{p}\right)$ of $\mathfrak{U}(n+1) \cap \mathfrak{B}^{\prime}$ such that $\mathfrak{B}_{1} \ni e_{t}{ }^{(n)}, t \in Z_{p}$. Let $\mathfrak{B}(n)$ be the 
algebra generated by $\mathfrak{B}$ and $\mathfrak{B}_{1}$. Then $\mathfrak{U}(n)^{\alpha} \subset \mathfrak{B}(n) \subset \mathfrak{U}(n+1)^{\alpha}$ and $\mathfrak{B}(n)$ is a type $I_{p^{n}}$ factor. Thus $\overline{U \mathfrak{B}(n)}=\overline{\cup \mathfrak{U}(n)^{\alpha}}=\mathfrak{U}^{\alpha}$ which completes the proof.

Remark 4.5. There is an example ( $\mathfrak{H}, \alpha)$ where $\mathfrak{U}^{\alpha}$ is not a UHF algebra but has a unique tracial state.

Let $\mathfrak{U}_{n}$ be a type $I_{p+1}$ factor and let $\alpha_{n}$ be the automorphism of $\mathfrak{A}_{n}$ implemented by $\exp \left\{2 \pi i p^{-1} \Sigma_{1}{ }^{p+1} k e_{k}\right\}$ where $\left\{e_{k}\right\}_{1}{ }^{p+1}$ is a family of orthogonal projections of $\mathfrak{A}_{n}$. We consider the system $\left(\mathfrak{H}=\bigotimes \mathfrak{U}_{n}, \alpha=\bigotimes \alpha_{n}\right)$. Then $\left(\mathfrak{H}, a^{\prime}\right)$ satisfies Condition 3.2 but $\mathfrak{H}$ does not contain a UHF subalgebra of type $\left(p^{n}\right)$. By Theorems 3.10 and 4.4 this proves our assertion.

\section{Acknowledgements}

The author would like to express his gratitude to Professor M. Takesaki and Dr. T. Hamachi. Their talks on AF algebras (at RIMS, July 1976) were very helpful. He would also like to thank Professor H. Araki and Dr. H. Takai for helpful discussions.

\section{References}

[1] Bratteli, O., Inductive limits of finile dimensional $\mathrm{C}^{*}$-algebras, Trans. Amer. Math. Soc., 171 (1972), 195-234.

[2] Connes, A., Une classification des lacteurs de type III, Ann. scient. Éc. Norm. Sup., $4^{e}$ série, 6 (1973), 133-252.

[3] Glimm, J. G., On a certain class of operator algebras, Trans. Amer. Math. Soc., 95 (1960), 318-340.

[4] Kishimoto, A. and Takai, H., On the invariant $\Gamma(\alpha)$ in $\mathrm{C}^{*}$-dynamical systems, Tóhoku Math. J. (to appear).

[5] Olesen, D., Inner *-automorphisms of simple C*-algebras, Comm. Math. Phys., 44 (1975), 175-190.

[6] Olesen, D., Pedersen, G. K. and St $\phi$ rmer, E., Compact abelian groups of automorphisms of simple C*-algebras, Inventiones Math., 39 (1977), 55-64.

[7] Stprmer, E., The even CAR algebra, Comm. Math. Phys., 16 (1970), 136-137. 
